Supporting Information for

\title{
Rational Design of Methodology-Independent Metal Parameters Using a Nonbonded Dummy Model
}

\author{
Yang Jiang ${ }^{\dagger}$, Haiyang Zhang ${ }^{\ddagger}$, and Tianwei Tan ${ }^{\dagger}$
}

${ }^{\dagger}$ Beijing Key Lab of Bioprocess, College of Life Science and Technology, Beijing University of Chemical Technology, Beijing 100029, China

${ }^{ \pm}$Department of Biological Science and Engineering, School of Chemistry and Biological Engineering, University of Science and Technology Beijing, 100083 Beijing, China

*Corresponding author:twtan@mail.buct.edu.cn 


\section{Contents}

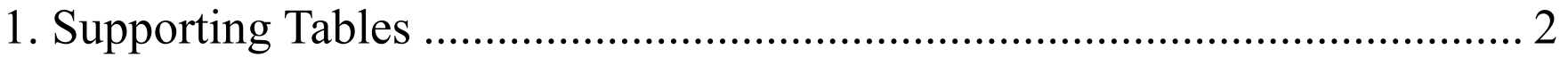



3. Example for Model Preparation Files........................................................... 18

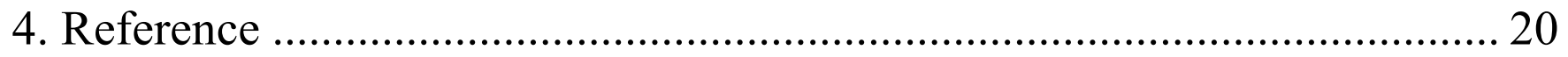




\section{Supporting Tables}

Table S1. Experimental values of divalent metal ion solvation free energy $(\mathrm{kcal} / \mathrm{mol})$ from Noyes ${ }^{1}$, Rosseinsky $^{2}$ and Marcus $^{3}$ with their "conventional" values ${ }^{a}$; our revised values ${ }^{b}$; experimental IOD values $(\AA)$ and $\mathrm{CN}$ values.

\begin{tabular}{|c|c|c|c|c|c|c|c|c|c|}
\hline $\begin{array}{l}\text { Divalent } \\
\text { cations }\end{array}$ & $\begin{array}{l}\text { Noyes } \\
\Delta \mathrm{G}_{\text {sol }}^{\text {exp }}\end{array}$ & $\begin{array}{c}\text { Rosseinsky } \\
\Delta \mathrm{G}_{\mathrm{sol}}^{\text {exp }}\end{array}$ & $\begin{array}{c}\text { Marcus } \\
\Delta \mathrm{G}_{\mathrm{sol}}^{\text {exp }}\end{array}$ & $\begin{array}{c}\text { Noyes } \\
\Delta \mathrm{G}_{\text {sol }}^{\text {conv }}\end{array}$ & $\begin{array}{c}\text { Rosseinsky } \\
\Delta \mathrm{G}_{\text {sol }}^{\text {conv }}\end{array}$ & $\begin{array}{l}\text { Marcus } \\
\Delta \mathrm{G}_{\text {sol }}^{\text {conv }}\end{array}$ & $\begin{array}{c}\text { Revised } \\
\Delta \mathrm{G}_{\text {sol }}^{\text {exp }}\end{array}$ & IOD & $\mathrm{CN}$ \\
\hline $\mathrm{Be}^{2+}$ & -582.3 & - & -572.1 & -61.3 & - & -69.5 & -603.2 & $1.670^{c}$ & $4^{c}$ \\
\hline $\mathrm{Mg}^{2+} *$ & -454.2 & -455.5 & -437.2 & 66.8 & 65.5 & 65.5 & -468.2 & $2.090 \pm 0.041^{c}$ & $6^{c}$ \\
\hline $\mathrm{Ca}^{2+}$ & -379.5 & -380.8 & -359.5 & 141.5 & 140.2 & 143.1 & -390.6 & $2.460^{c}$ & $8^{c}$ \\
\hline $\mathrm{Sr}^{2+}$ & -339.7 & -345.9 & -329.7 & 181.3 & 175.1 & 173.0 & -360.7 & $2.640 \pm 0.040^{c}$ & $8-15^{c}$ \\
\hline $\mathrm{Ba}^{2+}$ & -314 & -315.1 & -298.6 & 207 & 205.9 & 204.0 & -329.7 & $2.830^{d}$ & $9^{d}$ \\
\hline $\mathrm{Ra}^{2+}$ & -306 & - & -298.6 & 215 & - & 204.0 & -329.7 & - & - \\
\hline $\mathrm{V}^{2+} *$ & -441 & - & -436 & 80 & - & 66.7 & -467.0 & $2.210^{e}$ & $6^{e}$ \\
\hline $\mathrm{Cr}^{2+} *$ & -444.8 & - & -441.9 & 76.2 & - & 60.7 & -473.0 & Eq: $2.080^{f}$ & $6^{f}$ \\
\hline $\mathrm{Mn}^{2+} *$ & -436.4 & -437.8 & -420.4 & 84.6 & 83.2 & 82.2 & -451.5 & $2.192 \pm 0.013^{c}$ & $6^{c}$ \\
\hline $\mathrm{Fe}^{2+} *$ & -451.8 & -456.4 & -439.6 & 69.2 & 64.6 & 63.1 & -470.6 & $2.114 \pm 0.010^{c}$ & $6^{c}$ \\
\hline $\mathrm{Co}^{2+} *$ & -481 & -479.5 & -457.5 & 40 & 41.5 & 45.2 & -488.5 & $2.106 \pm 0.022^{c}$ & $6^{c}$ \\
\hline $\mathrm{Ni}^{2+} *$ & -492.8 & -494.2 & -473 & 28.2 & 26.8 & 29.6 & -504.1 & $2.061 \pm 0.014^{c}$ & $6^{c}$ \\
\hline $\mathrm{Cu}^{2+}$ & -496.2 & -498.7 & -480.2 & 24.8 & 22.3 & 22.5 & -511.2 & Avg: $2.110^{c}$ & $6^{c}$ \\
\hline $\mathrm{Zn}^{2+} *$ & -483.3 & -484.6 & -467 & 37.7 & 36.4 & 35.6 & -498.1 & $2.098 \pm 0.066^{c}$ & $6^{c}$ \\
\hline $\mathrm{Pd}^{2+}$ & -573 & - & -456.3 & -52 & - & 46.4 & -487.3 & - & - \\
\hline $\mathrm{Ag}^{2+}$ & - & - & -441.9 & - & - & 60.7 & -473.0 & - & - \\
\hline $\mathrm{Cd}^{2+} *$ & -429.1 & -430.5 & -419.3 & 91.88 & 90.5 & 83.4 & -450.3 & $2.301 \pm 0.025^{c}$ & $6^{c}$ \\
\hline $\mathrm{Sn}^{2+} *$ & -371.4 & - & -355.9 & 149.6 & - & 146.7 & -387.0 & Avg: $2.620^{c}$ & $6^{f}$ \\
\hline $\mathrm{Pt}^{2+}$ & - & - & -468.2 & - & - & 34.4 & -499.3 & - & - \\
\hline $\mathrm{Hg}^{2+} *$ & -434.9 & -436.3 & -420.4 & 86.07 & 84.7 & 82.2 & -451.5 & $2.410^{c}$ & $6^{c}$ \\
\hline $\mathrm{Pb}^{2+}$ & -356.5 & -357.8 & -340.4 & 164.47 & 163.2 & 162.2 & -371.5 & - & - \\
\hline $\mathrm{Sm}^{2+}$ & - & - & -328.5 & - & - & 174.2 & -359.5 & - & - \\
\hline $\mathrm{Eu}^{2+}$ & - & - & -330.9 & - & - & 171.8 & -361.9 & - & - \\
\hline $\mathrm{Yb}^{2+}$ & - & - & -360.7 & - & - & 141.9 & -391.8 & - & - \\
\hline
\end{tabular}

* The revised $\Delta G_{\text {sol }}^{\text {exp }}$ of the marked metal cations were used to generate our dummy atom models. ${ }^{a}$ The "conventional" values from Rosseinsky were obtained from the original paper ${ }^{2}$. The others were 
calculated by Eq. 3, where $\Delta G_{H}=-260.5 \mathrm{kcal} / \mathrm{mol}$ for Noyes and $-252.3 \mathrm{kcal} / \mathrm{mol}$ for Marcus. ${ }^{b}$ The revised values were calculated using Eq. 4. ${ }^{c}$ From Marcus's work ${ }^{4} .{ }^{d}$ From Smirnov's work ${ }^{5}{ }^{e}$ From Miyanaga's work ${ }^{6}{ }^{f}$ From Ohtaki's work ${ }^{7}$.

Table S2. Bond length, partial charge and quadrupole-moment trace $\gamma$ for TIP3P, SPEC/E and TIP4P-EW water models used in this work

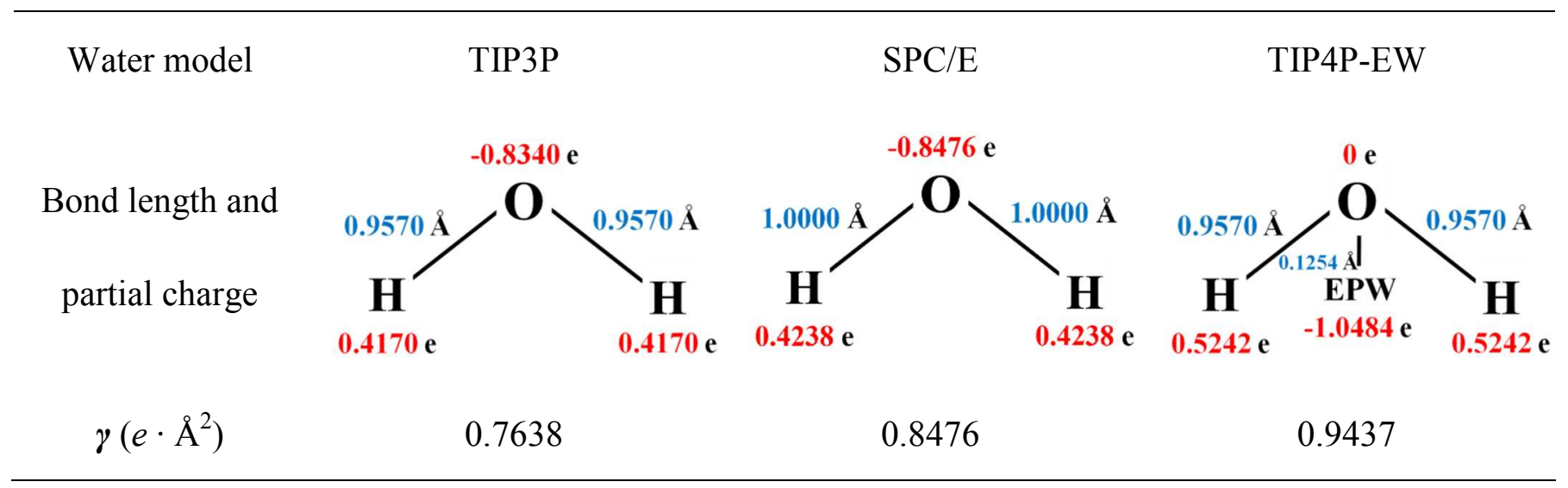

Table S3a. Calculated solvation free energies (corrected) of the parameter space for TIP3P water

\begin{tabular}{|c|c|c|c|c|c|c|c|}
\hline & & & & & & & \\
\hline
\end{tabular}

Table S3b. Calculated solvation free energies (corrected) of the parameter space for SPC/E water 


\begin{tabular}{|c|c|c|c|c|c|c|c|}
\hline & & & & & & & \\
\hline $\mathrm{R}(\AA)$ & 200 & 100 & 10 & 1 & 0.1 & 0.01 & 0.001 \\
\hline 0.30 & & & & & & & \\
\hline 0.50 & -525.0 & -516.9 & -493.9 & -494.4 & -496.7 & -496.0 & -495.3 \\
\hline 0.75 & -468.3 & -402.0 & -494.6 & -485.8 & -490.1 & -494.5 & -496.2 \\
\hline 1.00 & -433.6 & -420.8 & -466.0 & -478.2 & -487.4 & -486.6 & -492.7 \\
\hline 1.25 & -406.6 & -388.0 & -378.6 & -403.6 & -443.8 & -470.9 & -480.8 \\
\hline 1.50 & -388.0 & -365.1 & -344.4 & -362.5 & -402.7 & -446.8 & -473.4 \\
\hline
\end{tabular}

Table S3c. Calculated solvation free energies (corrected) of the parameter space for TIP4P-EW water

\begin{tabular}{|c|c|c|c|c|c|c|c|}
\hline & & & & & & & \\
\hline & & 100 & 10 & 1 & 0.1 & 0.01 & 0.001 \\
\hline 0.30 & -507.9 & -498.8 & -476.5 & -475.4 & -478.9 & -472.6 & -472.8 \\
\hline 0.50 & -493.6 & -484.4 & -474.5 & -471.7 & -473.1 & -467.6 & -478.0 \\
\hline 0.75 & -458.1 & -447.0 & -450.0 & -460.7 & -466.8 & -469.5 & -475.4 \\
\hline 1.00 & -430.1 & -414.8 & -407.0 & -432.9 & -455.1 & -464.4 & -469.5 \\
\hline 1.25 & -407.7 & -385.6 & -371.9 & -391.4 & -427.6 & -452.8 & -466.8 \\
\hline 1.50 & -390.2 & -365.1 & -340.2 & -356.5 & -390.3 & -429.2 & -454.2 \\
\hline
\end{tabular}

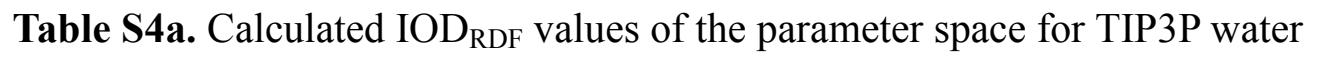

\begin{tabular}{|c|c|c|c|c|c|c|c|}
\hline $\mathrm{R}(\AA)$ & 200 & 100 & 10 & 1 & 0.1 & 0.01 & 0.001 \\
\hline 0.30 & & & & & & & \\
\hline 0.50 & 2.028 & 2.022 & 2.000 & 1.978 & 1.946 & 1.937 & 1.934 \\
\hline 0.75 & 2.355 & 2.100 & 2.058 & 2.021 & 1.983 & 1.952 & 1.939 \\
\hline 1.00 & 2.598 & 2.550 & 2.141 & 2.080 & 2.037 & 1.993 & 1.957 \\
\hline 1.25 & 2.885 & 2.804 & 2.589 & 2.159 & 2.086 & 2.041 & 1.999 \\
\hline 1.50 & 3.112 & 3.067 & 2.831 & 2.580 & 2.284 & 2.146 & 2.080 \\
\hline
\end{tabular}






\begin{tabular}{|c|c|c|c|c|c|c|c|}
\hline & 200 & 100 & 10 & 1 & 0.1 & 0.01 & 0.001 \\
\hline 0.30 & & & & & & & \\
\hline 0.50 & 2.036 & 2.031 & 2.010 & 1.992 & 1.971 & 1.960 & 1.960 \\
\hline 0.75 & 2.121 & 2.104 & 2.064 & 2.031 & 2.002 & 1.978 & 1.965 \\
\hline 1.00 & 2.355 & 2.255 & 2.146 & 2.085 & 2.045 & 2.012 & 1.980 \\
\hline 1.25 & 2.805 & 2.553 & 2.311 & 2.162 & 2.092 & 2.048 & 2.019 \\
\hline 1.50 & 3.125 & 2.810 & 2.586 & 2.306 & 2.162 & 2.091 & 2.050 \\
\hline
\end{tabular}

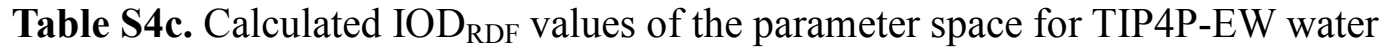

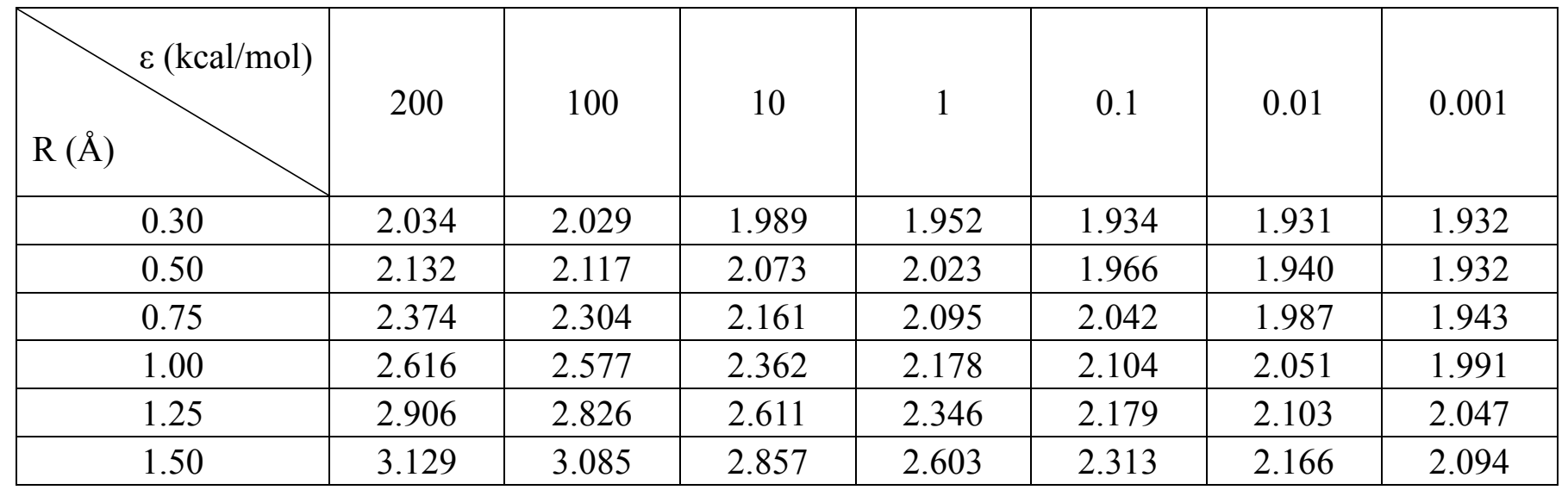

Table S5a. Calculated CN values of the parameter space for TIP3P water

\begin{tabular}{|c|c|c|c|c|c|c|c|}
\hline & 200 & 100 & 10 & 1 & 0.1 & 0.01 & 0.001 \\
\hline 0.30 & & & & & & & \\
\hline 0.50 & 6.0 & 6.0 & 6.0 & 6.0 & 6.0 & 6.0 & 6.0 \\
\hline 0.75 & 6.0 & 6.0 & 6.0 & 6.0 & 6.0 & 6.0 & 6.0 \\
\hline 1.00 & 9.9 & 6.9 & 6.0 & 6.0 & 6.0 & 6.0 & 6.0 \\
\hline 1.25 & 11.6 & 8.7 & 6.7 & 6.0 & 6.0 & 6.0 & 6.0 \\
\hline
\end{tabular}




\begin{tabular}{|l|l|l|l|l|l|l|l|}
\hline 1.50 & 12.0 & 11.8 & 9.3 & 8.0 & 6.1 & 6.0 & 6.0 \\
\hline
\end{tabular}

Table S5b. Calculated CN values of the parameter space for SPC/E water

\begin{tabular}{|c|c|c|c|c|c|c|c|}
\hline $\mathrm{R}(\AA)$ & 200 & 100 & 10 & 1 & 0.1 & 0.01 & 0.001 \\
\hline 0.30 & & & & & & & \\
\hline 0.50 & 6.0 & 6.0 & 6.0 & 6.0 & 6.0 & 6.0 & 6.0 \\
\hline 0.75 & 7.8 & 6.0 & 6.0 & 6.0 & 6.0 & 6.0 & 6.0 \\
\hline 1.00 & 8.9 & 8.5 & 6.0 & 6.0 & 6.0 & 6.0 & 6.0 \\
\hline 1.25 & 10.6 & 9.8 & 8.0 & 6.1 & 6.0 & 6.0 & 6.0 \\
\hline 1.50 & 12.0 & 11.5 & 9.0 & 7.9 & 6.0 & 6.0 & 6.0 \\
\hline
\end{tabular}

Table S5c. Calculated CN values of the parameter space for TIP4P-EW water

\begin{tabular}{|c|c|c|c|c|c|c|c|}
\hline & 200 & 100 & 10 & 1 & 0.1 & 0.01 & 0.001 \\
\hline 0.30 & & & & & & & \\
\hline 0.50 & 6.0 & 6.0 & 6.0 & 6.0 & 6.0 & 6.0 & 6.0 \\
\hline 0.75 & 8.0 & 6.0 & 6.0 & 6.0 & 6.0 & 6.0 & 6.0 \\
\hline 1.00 & 9.1 & 9.0 & 8.0 & 6.0 & 6.0 & 6.0 & 6.0 \\
\hline 1.25 & 11.9 & 11.7 & 10.0 & 8.0 & 6.0 & 6.0 & 6.0 \\
\hline 1.50 & 12.6 & 12.0 & 11.8 & 9.3 & 8.0 & 6.0 & 6.0 \\
\hline
\end{tabular}


Table S6. The reproduced coordination geometries and solvation free energies ${ }^{a}$ of models for the eleven divalent metal ions selected from the parameter generation process for SPC/E water (parameters with bad performances were marked in bold and gray)

\begin{tabular}{|c|c|c|c|c|c|c|c|c|c|c|}
\hline $\begin{array}{l}\text { Metal } \\
\text { ions }\end{array}$ & $\begin{array}{c}\text { Revised } \\
\Delta G_{\text {sol }}^{\text {exp }} \\
(\mathrm{kcal} / \mathrm{mol})\end{array}$ & $\begin{array}{c}\mathrm{IOD}^{\exp } \\
(\AA)\end{array}$ & $\mathrm{CN}$ & $\begin{array}{c}\mathrm{IOD}_{\mathrm{RDF}} \\
(\AA)\end{array}$ & $\mathrm{CN}_{\mathrm{RDF}}$ & $\begin{array}{l}\mathrm{R}_{\mathrm{I}} \\
(\AA)\end{array}$ & $\begin{array}{l}\Delta G_{M^{0} \rightarrow M^{2+}}^{T I} \\
(\mathrm{kcal} / \mathrm{mol})\end{array}$ & $\begin{array}{c}\Delta G_{v d W}^{S C} \\
(\mathrm{kcal} / \mathrm{mol})\end{array}$ & $\begin{array}{c}\Delta G_{\text {corr }} \\
(\mathrm{kcal} / \mathrm{mol})\end{array}$ & $\begin{array}{c}\Delta G_{\text {sol }}^{\text {cal }} \\
(\mathrm{kcal} / \mathrm{mol})\end{array}$ \\
\hline $\mathrm{Mg}^{2+}$ & -468.2 & 2.090 & 6 & 2.088 & 6.0 & 1.96 & -435.73 & 1.88 & -37.78 & -471.6 \\
\hline $\mathrm{V}^{2+}$ & -467.0 & 2.210 & 6 & 2.207 & 6.0 & 2.09 & -407.47 & -23.43 & -37.74 & -468.6 \\
\hline $\mathrm{Cr}^{2+}$ & -473.0 & 2.080 & 6 & 2.079 & 6.0 & 1.92 & -438.52 & 1.58 & -37.80 & -474.7 \\
\hline $\mathrm{Mn}^{2+}$ & -451.5 & 2.192 & 6 & 2.193 & 6.0 & 2.06 & -406.19 & -8.43 & -37.74 & -452.4 \\
\hline $\mathrm{Fe}^{2+}$ & -470.6 & 2.114 & 6 & 2.109 & 6.0 & 1.97 & -431.18 & -0.94 & -37.78 & -469.9 \\
\hline $\mathrm{Co}^{2+}$ & -488.5 & 2.106 & 6 & 2.107 & 6.0 & 1.99 & -440.24 & -10.93 & -37.77 & -488.9 \\
\hline $\mathrm{Ni}^{2+}$ & -504.1 & 2.061 & 6 & 2.054 & 6.0 & 1.90 & -456.31 & -8.30 & -37.80 & -502.4 \\
\hline $\mathrm{Zn}^{2+}$ & -498.1 & 2.098 & 6 & 2.093 & 6.0 & 1.98 & -446.08 & -13.77 & -37.77 & -497.6 \\
\hline $\mathrm{Cd}^{2+}$ & -450.3 & 2.301 & 6 & 2.284 & 6.4 & 2.13 & -388.20 & -23.73 & -37.72 & -449.7 \\
\hline $\mathrm{Sn}^{2+}$ & -387.0 & 2.620 & 6 & 2.595 & 8.2 & 2.40 & -332.80 & -16.86 & -37.60 & -387.3 \\
\hline $\mathrm{Hg}^{2+}$ & -451.5 & 2.410 & 6 & 2.401 & 7.8 & 2.22 & -377.46 & -36.48 & -37.68 & -451.6 \\
\hline
\end{tabular}

${ }^{a}$ The detail values of free energy calculation and correction are shown in the table. $\mathrm{R}_{\mathrm{I}}$ is the ionic radius that is derived from the RDF plot and used to calculate the free energy correction. $\Delta G_{c o r r}$ is the sum of $\Delta G_{\text {corr }}^{P B C}$ and $\Delta G_{\text {corr }}^{\phi}$ calculated using Eq. 5 and 6. 
Table S7. The reproduced coordination geometries and solvation free energies ${ }^{a}$ of models for the eleven divalent metal ions selected from the parameter generation process for TIP4P-EW water (parameters with bad performances were marked in bold and gray)

\begin{tabular}{|c|c|c|c|c|c|c|c|c|c|c|}
\hline $\begin{array}{l}\text { Metal } \\
\text { ions }\end{array}$ & $\begin{array}{c}\text { Revised } \\
\Delta G_{\text {sol }}^{\text {exp }} \\
(\mathrm{kcal} / \mathrm{mol})\end{array}$ & $\begin{array}{c}\mathrm{IOD}^{\exp } \\
(\AA)\end{array}$ & $\mathrm{CN}$ & $\begin{array}{c}\mathrm{IOD}_{\mathrm{RDF}} \\
(\AA)\end{array}$ & $\mathrm{CN}_{\mathrm{RDF}}$ & $\begin{array}{l}\mathrm{R}_{\mathrm{I}} \\
(\AA)\end{array}$ & $\begin{array}{l}\Delta G_{M^{0} \rightarrow M^{2+}}^{T I} \\
(\mathrm{kcal} / \mathrm{mol})\end{array}$ & $\begin{array}{c}\Delta G_{v d W}^{S C} \\
(\mathrm{kcal} / \mathrm{mol})\end{array}$ & $\begin{array}{c}\Delta G_{\text {corr }} \\
(\mathrm{kcal} / \mathrm{mol})\end{array}$ & $\begin{array}{c}\Delta G_{\text {sol }}^{\text {cal }} \\
(\mathrm{kcal} / \mathrm{mol})\end{array}$ \\
\hline $\mathrm{Mg}^{2+}$ & -468.2 & 2.090 & 6 & 2.092 & 6.0 & 1.94 & -420.29 & -3.19 & -42.25 & -465.7 \\
\hline $\mathrm{V}^{2+}$ & -467.0 & 2.210 & 6 & 2.184 & 6.0 & 2.05 & -400.09 & -26.10 & -42.21 & -468.4 \\
\hline $\mathrm{Cr}^{2+}$ & -473.0 & 2.080 & 6 & 2.079 & 6.0 & 1.93 & -425.89 & -4.37 & -42.25 & -472.5 \\
\hline $\mathrm{Mn}^{2+}$ & -451.5 & 2.192 & 6 & 2.189 & 6.0 & 2.06 & -395.95 & -15.34 & -42.20 & -453.5 \\
\hline $\mathrm{Fe}^{2+}$ & -470.6 & 2.114 & 6 & 2.109 & 6.0 & 1.96 & -419.80 & -8.64 & -42.24 & -470.7 \\
\hline $\mathrm{Co}^{2+}$ & -488.5 & 2.106 & 6 & 2.106 & 6.0 & 1.99 & -425.70 & -20.70 & -42.23 & -488.6 \\
\hline $\mathrm{Ni}^{2+}$ & -504.1 & 2.061 & 6 & 2.088 & 6.0 & 1.95 & -434.41 & -26.74 & -42.25 & -503.4 \\
\hline $\mathrm{Zn}^{2+}$ & -498.1 & 2.098 & 6 & 2.108 & 6.0 & 1.99 & -427.22 & -28.63 & -42.23 & -498.1 \\
\hline $\mathrm{Cd}^{2+}$ & -450.3 & 2.301 & 6 & 2.296 & 6.9 & 2.15 & -380.32 & -28.93 & -42.17 & -451.4 \\
\hline $\mathrm{Sn}^{2+}$ & -387.0 & 2.620 & 6 & 2.603 & 8.6 & 2.40 & -327.20 & -19.41 & -42.06 & -388.7 \\
\hline $\mathrm{Hg}^{2+}$ & -451.5 & 2.410 & 6 & 2.393 & 8.0 & 2.23 & -370.74 & -40.21 & -42.13 & -453.1 \\
\hline
\end{tabular}

${ }^{a}$ The detail values of free energy calculation and correction are shown in the table. $\mathrm{R}_{\mathrm{I}}$ is the ionic radius that is derived from the RDF plot and used to calculate the free energy correction. $\Delta G_{c o r r}$ is the sum of $\Delta G_{\text {corr }}^{P B C}$ and $\Delta G_{\text {corr }}^{\phi}$ calculated using Eq. 5 and 6. 
Table S8a. Parameter refining for $\mathrm{V}^{2+}$ with TIP4P-EW water

\begin{tabular}{|c|c|c|c|c|}
\hline \multirow[b]{2}{*}{ Parameter combination } & \multicolumn{4}{|c|}{ Eligible models after Step 1} \\
\hline & $\begin{array}{c}R=0.6360 \\
\varepsilon=144.5440\end{array}$ & $\begin{array}{c}R=0.6400 \\
\varepsilon=155.5966\end{array}$ & $\begin{array}{c}R=0.6440 \\
\varepsilon=141.9058\end{array}$ & $\begin{array}{c}R=0.6440 \\
\varepsilon=147.2313\end{array}$ \\
\hline & \multicolumn{4}{|c|}{ Eligible models after Step 2} \\
\hline Parameter combination & \multicolumn{4}{|c|}{$\begin{array}{c}R=0.6360 \\
\varepsilon=144.5440\end{array}$} \\
\hline $\operatorname{IOD}_{\mathrm{RDF}}(\AA)$ & \multicolumn{4}{|c|}{$2.202(0.36 \%)^{a}$} \\
\hline \multirow[t]{2}{*}{$\mathrm{CN}$} & \multicolumn{4}{|c|}{6.0} \\
\hline & \multicolumn{4}{|c|}{ Eligible models after Step 3} \\
\hline Parameter combination & \multicolumn{4}{|c|}{$\begin{array}{c}R=0.6360 \\
\varepsilon=144.5440\end{array}$} \\
\hline$\Delta G_{\text {sol }}^{\text {cal }}(\mathrm{kcal} / \mathrm{mol})$ & \multicolumn{4}{|c|}{$-469.2(0.46 \%)^{b}$} \\
\hline
\end{tabular}

${ }^{a}$ The relative error of the calculated $\operatorname{IOD}_{\mathrm{RDF}}$ value with the experimental value $(2.210 \AA)$ as the standard.

${ }^{b}$ The relative error of the calculated $\Delta G_{\text {sol }}^{\text {cal }}$ value with the experimental value $(-467.0 \mathrm{kcal} / \mathrm{mol})$ as the standard. 
Table S8b. Parameter refining for $\mathrm{Ni}^{2+}$ with TIP4P-EW water

\begin{tabular}{|c|c|}
\hline & Eligible models after Step 1 \\
\hline \multirow[t]{2}{*}{ Parameter combination } & $\begin{array}{c}R=0.3280 \\
\varepsilon=201.3724\end{array}$ \\
\hline & Eligible models after Step 2 \\
\hline Parameter combination & $\begin{array}{c}R=0.3280 \\
\varepsilon=201.3724\end{array}$ \\
\hline $\operatorname{IOD}_{\mathrm{RDF}}(\AA)$ & $2.051(0.49 \%)^{a}$ \\
\hline \multirow[t]{2}{*}{$\mathrm{CN}$} & 6.0 \\
\hline & Eligible models after Step 3 \\
\hline Parameter combination & $\begin{array}{c}R=0.3280 \\
\varepsilon=201.3724\end{array}$ \\
\hline$\Delta G_{\text {sol }}^{\text {cal }}(\mathrm{kcal} / \mathrm{mol})$ & $-506.8(0.54 \%)^{b}$ \\
\hline
\end{tabular}

${ }^{a}$ The relative error of the calculated $\operatorname{IOD}_{\mathrm{RDF}}$ value with the experimental value $(2.061 \AA)$ as the standard.

${ }^{b}$ The relative error of the calculated $\Delta G_{\text {sol }}^{\text {cal }}$ value with the experimental value $(-504.1 \mathrm{kcal} / \mathrm{mol})$ as the standard. 
Table S9a. Calculated geometry ${ }^{a}$ of the $\mathrm{Ni}^{2+}$ center of $E$. coli GlxI compared with the corresponding crystal structure (PDB ID: 1F9Z) ${ }^{8}$

\begin{tabular}{lllllll}
\hline & Glu56 $\mathrm{O}^{\varepsilon}$ & Glu122 $\mathrm{O}^{\varepsilon}$ & His5 $\mathrm{N}^{\varepsilon}$ & His74 $\mathrm{N}^{\varepsilon}$ & Water1 & Water2 \\
\cline { 2 - 6 } $\mathrm{Ni}_{\mathrm{A} \text {, present }}$ & $1.98 \pm 0.04$ & $1.98 \pm 0.04$ & $2.13 \pm 0.05$ & $2.15 \pm 0.05$ & $2.09 \pm 0.04$ & $2.09 \pm 0.04$ \\
$\mathrm{Ni}_{\mathrm{A} \text {, Duarte }}$ & $1.99 \pm 0.03$ & $1.99 \pm 0.03$ & $2.13 \pm 0.04$ & $2.17 \pm 0.05$ & $2.08 \pm 0.03$ & $2.08 \pm 0.04$ \\
$\mathrm{Ni}_{\mathrm{A}, \text { LM1264 }}$ & $2.00 \pm 0.04$ & $2.00 \pm 0.04$ & $2.16 \pm 0.06$ & $2.17 \pm 0.06$ & $2.09 \pm 0.05$ & $2.09 \pm 0.05$ \\
$\mathrm{Ni}_{\mathrm{A} \text {, exp }}$ & 2.1 & 2.1 & 2.2 & 2.3 & 2.1 & 2.2 \\
$\mathrm{Ni}_{\mathrm{B}, \text { present }}$ & $1.98 \pm 0.04$ & $1.98 \pm 0.04$ & $2.13 \pm 0.05$ & $2.14 \pm 0.05$ & $2.09 \pm 0.04$ & $2.08 \pm 0.04$ \\
$\mathrm{Ni}_{\mathrm{B}, \text { Duarte }}$ & $1.99 \pm 0.03$ & $1.99 \pm 0.03$ & $2.13 \pm 0.04$ & $2.17 \pm 0.05$ & $2.08 \pm 0.03$ & $2.08 \pm 0.04$ \\
$\mathrm{Ni}_{\mathrm{B}, \text { LM1264 }}$ & $2.00 \pm 0.04$ & $2.00 \pm 0.04$ & $2.15 \pm 0.05$ & $2.17 \pm 0.06$ & $2.09 \pm 0.05$ & $2.09 \pm 0.05$ \\
$\mathrm{Ni}_{\mathrm{B} \text {, exp }}$ & 2.1 & 2.1 & 2.1 & 2.3 & 2.1 & 2.2 \\
\hline
\end{tabular}

${ }^{a}$ The calculated geometries of Duarte's model $\left(\mathrm{Ni}_{\mathrm{A} \text { or B, Duarte }}\right)$ are obtained from Duarte's work ${ }^{9}$ and the calculated geometries of Li-Merz 12-6-4 $\operatorname{model}^{10}\left(\mathrm{Ni}_{\mathrm{A} \text { or B, LM1264 }}\right)$ and the present model $\left(\mathrm{Ni}_{\mathrm{A} \text { or B, present }}\right)$ are calculated by the method described in Section 2.7.

Table S9b. Calculated geometry ${ }^{a}$ of the $\mathrm{Co}^{2+}$ center of E. coli GlxI compared with the corresponding crystal structure (PDB ID: 1FA6) $^{8}$

\begin{tabular}{lllllll}
\hline & Glu56 $\mathrm{O}^{\varepsilon}$ & Glu122 $\mathrm{O}^{\varepsilon}$ & His5 $\mathrm{N}^{\varepsilon}$ & His74 $\mathrm{N}^{\varepsilon}$ & Water1 & Water2 \\
\cline { 2 - 6 } $\mathrm{Co}_{\mathrm{A}, \text { present }}$ & $2.03 \pm 0.03$ & $2.03 \pm 0.03$ & $2.18 \pm 0.04$ & $2.19 \pm 0.05$ & $2.12 \pm 0.04$ & $2.13 \pm 0.03$ \\
$\mathrm{Co}_{\mathrm{A} \text {, Duarte }}$ & $2.01 \pm 0.03$ & $2.01 \pm 0.03$ & $2.19 \pm 0.05$ & $2.27 \pm 0.09$ & $2.08 \pm 0.03$ & $2.11 \pm 0.04$ \\
\hline
\end{tabular}




\begin{tabular}{lllllll}
\hline $\mathrm{Co}_{\mathrm{A}, \text { LM1264 }}$ & $2.04 \pm 0.04$ & $2.04 \pm 0.04$ & $2.19 \pm 0.05$ & $2.21 \pm 0.06$ & $2.13 \pm 0.05$ & $2.13 \pm 0.05$ \\
$\mathrm{Co}_{\mathrm{A}, \text { exp }}$ & 2.1 & 2.2 & 2.3 & 2.4 & 2.3 & 2.4 \\
$\mathrm{Co}_{\mathrm{B}, \text { present }}$ & $2.03 \pm 0.03$ & $2.03 \pm 0.03$ & $2.19 \pm 0.05$ & $2.18 \pm 0.04$ & $2.13 \pm 0.04$ & $2.12 \pm 0.04$ \\
$\mathrm{Co}_{\mathrm{B}, \text { Duarte }}$ & $2.01 \pm 0.03$ & $2.01 \pm 0.03$ & $2.19 \pm 0.05$ & $2.27 \pm 0.09$ & $2.08 \pm 0.03$ & $2.11 \pm 0.04$ \\
$\mathrm{Co}_{\mathrm{B}, \text { LM} 1264}$ & $2.04 \pm 0.04$ & $2.04 \pm 0.04$ & $2.19 \pm 0.05$ & $2.20 \pm 0.06$ & $2.13 \pm 0.05$ & $2.12 \pm 0.05$ \\
$\mathrm{Co}_{\text {B, exp }}$ & 2.1 & 2.4 & 2.2 & 2.3 & 2.2 & 2.4 \\
\hline
\end{tabular}

${ }^{a}$ The calculated geometries of Duarte's model $\left(\mathrm{Co}_{\mathrm{A} \text { or B, Duarte }}\right)$ are obtained from Duarte's work ${ }^{9}$ and the calculated geometries of Li-Merz 12-6-4 $\operatorname{model}^{10}\left(\mathrm{Co}_{\mathrm{A} \text { or B, LM1264 }}\right)$ and the present model $\left(\mathrm{Co}_{\mathrm{A} \text { or B, present }}\right)$ are calculated by the method described in Section 2.7.

Table S9c. Calculated geometry ${ }^{a}$ of the $\mathrm{Cd}^{2+}$ center of E. coli GlxI compared with the corresponding crystal structure (PDB ID: 1FA7) ${ }^{8}$

\begin{tabular}{lllllllll}
\hline & Glu56 $^{\varepsilon 1}$ & Glu56 $\mathrm{O}^{\varepsilon 2}$ & Glu122 $\mathrm{O}^{\varepsilon 1}$ & Glu122 $\mathrm{O}^{\varepsilon 2}$ & His5 N & His74 $\mathrm{N}^{\varepsilon}$ & Water1 & Water2 \\
\cline { 2 - 9 } $\mathrm{Cd}_{\mathrm{A} \text {, present }}$ & $2.24 \pm 0.03$ & None & $2.24 \pm 0.03$ & None & $2.37 \pm 0.04$ & $2.38 \pm 0.05$ & $2.31 \pm 0.04$ & $2.31 \pm 0.04$ \\
$\mathrm{Cd}_{\mathrm{A}, \text { LM1264 }}$ & $2.71 \pm 0.65$ & $2.53 \pm 0.57$ & $2.20 \pm 0.07$ & $2.91 \pm 0.65$ & $2.33 \pm 0.08$ & $2.35 \pm 0.07$ & $2.27 \pm 0.07$ & $2.27 \pm 0.07$ \\
$\mathrm{Cd}_{\mathrm{A}, \text { LM1264 }}^{*}$ & $2.13 \pm 0.04$ & None & $2.13 \pm 0.05$ & None & $2.30 \pm 0.06$ & $2.32 \pm 0.06$ & $2.26 \pm 0.06$ & $2.25 \pm 0.06$ \\
$\mathrm{Cd}_{\mathrm{A} \text {, exp }}$ & 2.4 & None & 2.8 & None & 2.4 & 2.6 & 2.4 & 2.4 \\
$\mathrm{Cd}_{\mathrm{B} \text {, present }}$ & $2.24 \pm 0.03$ & None & $2.24 \pm 0.03$ & None & $2.37 \pm 0.04$ & $2.38 \pm 0.05$ & $2.31 \pm 0.04$ & $2.31 \pm 0.04$ \\
$\mathrm{Cd}_{\mathrm{B}, \text { LM1264 }}$ & $2.27 \pm 0.28$ & None & $2.44 \pm 0.48$ & $2.68 \pm 0.66$ & $2.33 \pm 0.08$ & $2.34 \pm 0.07$ & $2.27 \pm 0.07$ & $2.27 \pm 0.07$ \\
$\mathrm{Cd}_{\text {B, LM1264 }}^{*}$ & $2.14 \pm 0.05$ & None & $2.18 \pm 0.10$ & $2.90 \pm 0.63$ & $2.33 \pm 0.07$ & $2.33 \pm 0.06$ & $2.26 \pm 0.06$ & $2.28 \pm 0.07$ \\
$\mathrm{Cd}_{\mathrm{B} \text {, exp }}$ & 2.3 & None & 2.4 & None & 2.3 & 2.5 & 2.4 & 2.6 \\
\hline
\end{tabular}

${ }^{a}$ The calculated geometries of Duarte's model $\left(\mathrm{Cd}_{\mathrm{A} \text { or B, Duarte }}\right)$ are obtained from Duarte's work ${ }^{9}$ and the calculated geometries of Li-Merz 12-6-4 $\operatorname{model}^{10}\left(\mathrm{Cd}_{\mathrm{A} \text { or B, LM1264 }}\right)$ and the present model $\left(\mathrm{Cd}_{\mathrm{A} \text { or B, present }}\right)$ 
are calculated by the method described in Section 2.7. The Li-Merz 12-6-4 model with additional modification of the side chain charge of Glu residue is also tested $\left(\mathrm{Cd}^{*} \mathrm{~A}\right.$ or B, LM1264, according to the Duarte work ${ }^{9}$ ). There are additional ligands (e.g. Glu122 $\mathrm{O}^{\varepsilon 2}$ ) existing when using the Li-Merz 12-6-4 model, leading to the $\mathrm{CN}$ greater than six. "None" means that the corresponding atom does not coordinate to the metal ion.

Table S9d. Calculated geometry ${ }^{a}$ of the $\mathrm{Zn}^{2+}$ center of $E$. coli GlxI compared with the corresponding crystal structure (PDB ID: 1FA5) ${ }^{8}$

\begin{tabular}{lllllll}
\hline & Glu56 $\mathrm{O}^{\varepsilon}$ & Glu122 $\mathrm{O}^{\varepsilon}$ & His5 $\mathrm{N}^{\varepsilon}$ & His74 $\mathrm{N}^{\varepsilon}$ & Water1 & Water2 \\
\cline { 2 - 6 } $\mathrm{Zn}$ & $2.02 \pm 0.03$ & $2.02 \pm 0.03$ & $2.17 \pm 0.04$ & $2.18 \pm 0.05$ & $2.11 \pm 0.04$ & $2.12 \pm 0.04$ \\
$\mathrm{Zn}_{\text {A, Duarte }}$ & $2.01 \pm 0.03$ & $2.01 \pm 0.03$ & $2.18 \pm 0.04$ & $2.25 \pm 0.05$ & $2.08 \pm 0.04$ & $2.11 \pm 0.04$ \\
$\mathrm{Zn}_{\mathrm{A}, \text { LM1264 }}$ & $2.03 \pm 0.04$ & $2.03 \pm 0.04$ & $2.18 \pm 0.05$ & $2.20 \pm 0.06$ & $2.12 \pm 0.05$ & $2.12 \pm 0.05$ \\
$\mathrm{Zn}_{\mathrm{A} \text {, exp }}$ & 2.1 & 2.1 & 2.2 & 2.2 & 2.0 & Not exist \\
$\mathrm{Zn}_{\mathrm{B}, \text { present }}$ & $2.02 \pm 0.03$ & $2.02 \pm 0.03$ & $2.16 \pm 0.04$ & $2.18 \pm 0.05$ & $2.12 \pm 0.04$ & $2.11 \pm 0.04$ \\
$\mathrm{Zn}_{\mathrm{B}, \text { Duarte }}$ & $2.00 \pm 0.03$ & $2.01 \pm 0.03$ & $2.18 \pm 0.04$ & $2.25 \pm 0.05$ & $2.08 \pm 0.04$ & $2.11 \pm 0.04$ \\
$\mathrm{Zn}_{\mathrm{B}, \text { LM1264 }}$ & $2.03 \pm 0.05$ & $2.03 \pm 0.04$ & $2.18 \pm 0.06$ & $2.19 \pm 0.05$ & $2.12 \pm 0.05$ & $2.12 \pm 0.05$ \\
$\mathrm{Zn}_{\text {B, exp }}$ & 2.1 & 2.4 & 2.1 & 2.2 & 2.1 & Not exist \\
\hline
\end{tabular}

${ }^{a}$ The calculated geometries of Duarte's model $\left(\mathrm{Zn}_{\mathrm{A} \text { or B, Duarte }}\right)$ are obtained from Duarte's work ${ }^{9}$ and the calculated geometries of Li-Merz 12-6-4 $\operatorname{model}^{10}\left(\mathrm{Zn}_{\mathrm{A} \text { or B, LM1264 }}\right)$ and the present model $\left(\mathrm{Zn}_{\mathrm{A} \text { or B, present }}\right)$ are calculated by the method described in Section 2.7. "Not exist" means that, in the crystal structure with $\mathrm{Zn}^{2+}$, the metal ion coordinated with only one water molecule, leading the coordination number equal to five. 


\section{Supporting Figures}

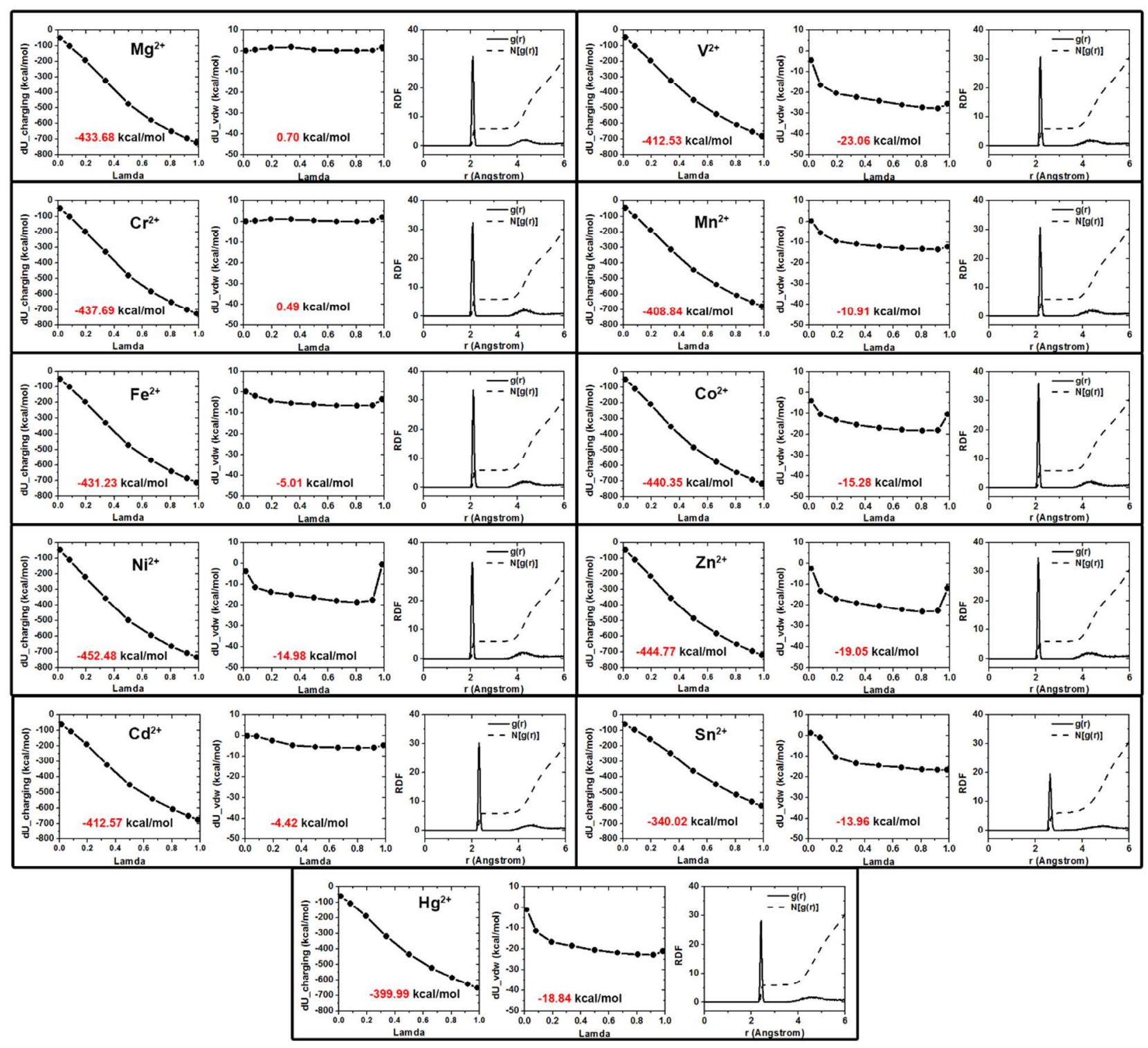

Figure S1. The dU/d $\lambda$ vs. $\lambda$ plot in the free energy calculation and the RDF plot with the molecule number curve of our final models for TIP3P water

In each subfigure surrounded by a black border, from left to right, the contents are the $\mathrm{dU} / \mathrm{d} \lambda$ plot for the charge perturbation, the $\mathrm{dU} / \mathrm{d} \lambda$ plot for the vdW perturbation and the RDF $(\mathrm{g}(\mathrm{r}))$ plot with the molecule 
number curve $(\mathrm{N}[\mathrm{g}(\mathrm{r})])$ of our final models for TIP3P water. The other systems show similar landscapes and we do not show them. The red numbers are the integral values for all the TI processes.

a)

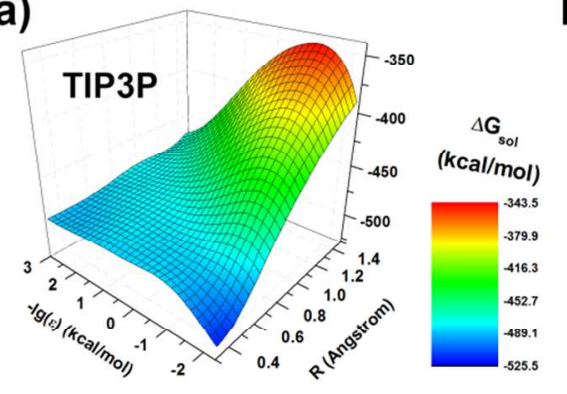

b)

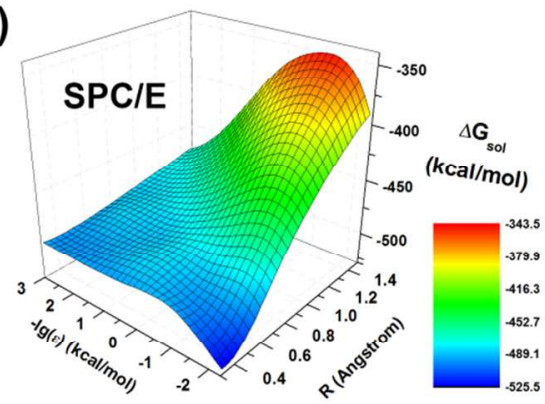

c)

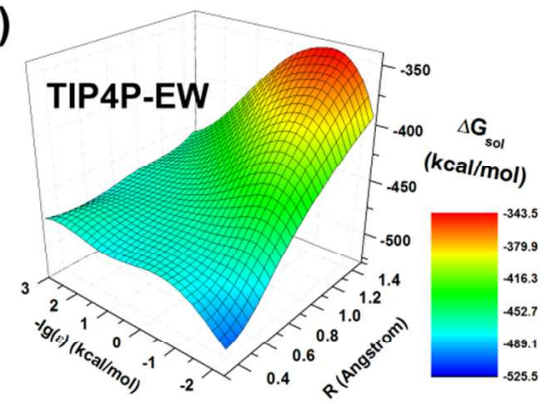

Figure S2. The solvation free energy surfaces within the parameter space for (a) TIP3P water, (b)

\section{SPC/E water and (c) TIP4P-EW water}

The free energy surfaces are colored from blue to red according their magnitude from low to high. The parameter space is shown as the $\mathrm{x}-\mathrm{y}$ plane within $R \in[0.3000,1.5000] \AA$ and $-\lg (\varepsilon) \in[-2.3010,3.0000]$ $\mathrm{kcal} / \mathrm{mol}$.
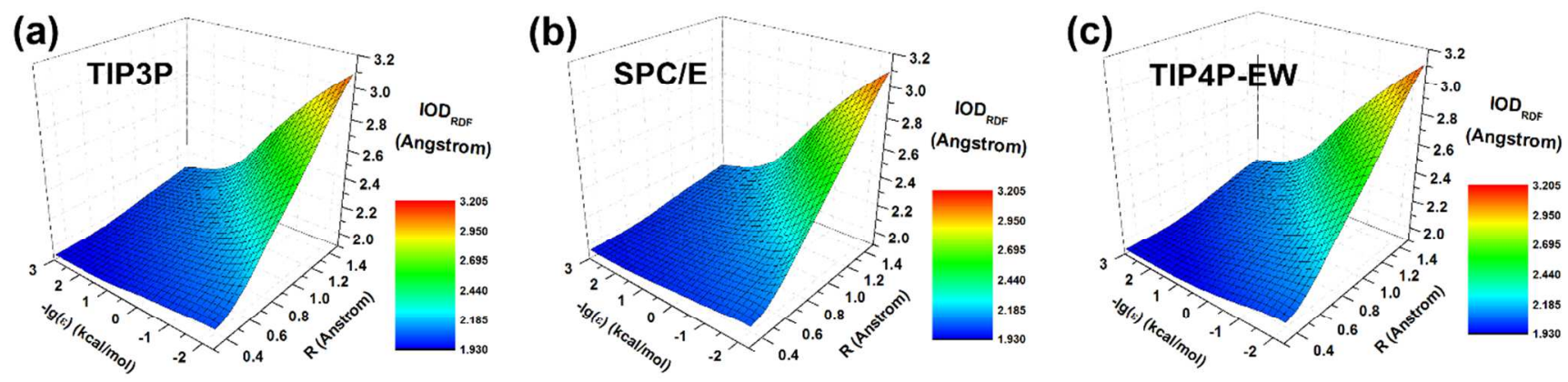

Figure S3. The IOD $\mathrm{RDF}$ surfaces within the parameter space for (a) TIP3P water, (b) SPC/E water and (c) TIP4P-EW water

The $\mathrm{IOD}_{\mathrm{RDF}}$ surfaces are colored from blue to red according their magnitude from low to high. The 
parameter space is shown as the $\mathrm{x}-\mathrm{y}$ plane within $R \in[0.3000,1.5000] \AA$ and $-\lg (\varepsilon) \in[-2.3010,3.0000]$ $\mathrm{kcal} / \mathrm{mol}$.

Figure S4. The solvation free energy contour curves with the IOD contour curves and their intersection points for (a) TIP3P water, (b) SPC/E water, and (c) TIP4P-EW water

The contour curves of the solvation free energy surface and the $\mathrm{IOD}_{\mathrm{RDF}}$ surface are shown as solid lines and dotted lines, respectively. The contour curves and their intersection point for the eleven metal ions are represented by different colors: magenta for $\mathrm{Mg}^{2+}$, yellow for $\mathrm{V}^{2+}$, blue for $\mathrm{Cr}^{2+}$, dark yellow for $\mathrm{Mn}^{2+}$, cyan for $\mathrm{Fe}^{2+}$, green for $\mathrm{Co}^{2+}$, black for $\mathrm{Ni}^{2+}$, red for $\mathrm{Zn}^{2+}$, violet for $\mathrm{Cd}^{2+}$, orange for $\mathrm{Sn}^{2+}$ and olive for $\mathrm{Hg}^{2+}$. Note that the solvation free energy contour curves of $\mathrm{Hg}^{2+}$ and $\mathrm{Mn}^{2+}$ are overlapped because of the same free energy values, so we only show the curve for $\mathrm{Mn}^{2+}$ with dark yellow. 


\section{Example for Model Preparation Files}

The ".prepi" files for all the models are similar with the following example for $\mathrm{Mg}^{2+}$. To create this file for another metal ion, please change the related residue name.

\section{Example for the .prepi file:}

\begin{tabular}{|c|c|c|c|c|c|c|c|c|c|c|}
\hline 0 & 0 & 2 & & & & & & & & \\
\hline \multicolumn{11}{|c|}{$\begin{array}{l}\text { This is a remark line } \\
\text { molecule.res }\end{array}$} \\
\hline MG2 & INT & 0 & & & & & \# MG2 c & an be chang & ed to anotl & her residue name. \\
\hline \multicolumn{2}{|c|}{ CORRECT } & \multicolumn{2}{|c|}{ OMIT DU } & \multicolumn{3}{|c|}{ BEG } & & & & \\
\hline \multicolumn{2}{|c|}{0.0000} & & & & & & & & & \\
\hline 1 & DUMM & DU & M & 0 & -1 & -2 & 0.000 & .0 & .0 & .00000 \\
\hline 2 & DUMM & DU & M & 1 & 0 & -1 & 1.449 & .0 & .0 & .00000 \\
\hline 3 & DUMM & DU & M & 2 & 1 & 0 & 1.522 & 111.1 & .0 & .00000 \\
\hline 4 & $\mathrm{MC}$ & $\mathrm{MC}$ & $\mathrm{M}$ & 3 & 2 & 1 & 1.540 & 111.208 & 180.000 & -1.000000 \\
\hline 5 & DU1 & D1 & $\mathrm{E}$ & 4 & 3 & 2 & 0.900 & 180.000 & 180.000 & 0.500000 \\
\hline 6 & DU2 & D2 & $\mathrm{E}$ & 4 & 3 & 2 & 0.900 & 90.000 & -0.000 & 0.500000 \\
\hline 7 & DU3 & D3 & $\mathrm{E}$ & 4 & 3 & 2 & 0.900 & 90.000 & 90.000 & 0.500000 \\
\hline 8 & DU4 & D1 & $\mathrm{E}$ & 4 & 3 & 2 & 0.900 & 0.000 & -0.000 & 0.500000 \\
\hline 9 & DU5 & D2 & $\mathrm{E}$ & 4 & 3 & 2 & 0.900 & -90.000 & -0.000 & 0.500000 \\
\hline 10 & DU6 & D3 & $\mathrm{E}$ & 4 & 3 & 2 & 0.900 & -90.000 & 90.000 & 0.500000 \\
\hline \multicolumn{11}{|l|}{ LOOP } \\
\hline \multicolumn{11}{|c|}{ IMPROPER } \\
\hline \multicolumn{11}{|l|}{ DONE } \\
\hline STOP & & & & & & & & & & \\
\hline
\end{tabular}

The "..frcmod" files for all the models are similar with the following example for $\mathrm{Mg}^{2+}$. To create this file for another metal ion, please change the mass and the nonbonding parameters of MC. All the parameters can be found in Table 1.

Example for the .frcmod file: 




D2 3.000

\# The mass of MC can be changed to that for another model.

D3 3.000

0.000

0.000

BOND

MC-D1 $800.0 \quad 0.900$

MC-D2 $800.0 \quad 0.900$

MC-D3 $\quad 800.0 \quad 0.900$

ANGLE

D1-MC-D1 $125.0 \quad 180.0$

D2-MC-D2 $\quad 125.0 \quad 180.0$

D3-MC-D3 $125.0 \quad 180.0$

D1-MC-D2 $\quad 125.0 \quad 90.0$

D1-MC-D3 $\quad 125.0 \quad 90.0$

D2-MC-D3 $\quad 125.0 \quad 90.0$

D2-MC-D1 $125.0 \quad 90.0$

D3-MC-D1 $125.0 \quad 90.0$

D3-MC-D2 $125.0 \quad 90.0$

DIHE

IMPROPER

NONBON

MC $\quad 0.9203 \quad 0.2394 \quad$ \# Rmin/2 and epsilon can be changed to those for another model.

$\begin{array}{lll}\mathrm{D} 1 & 1.3882 & 0.00000001\end{array}$

$\begin{array}{lll}\mathrm{D} 2 & 1.3882 & 0.00000001\end{array}$

D3 $\quad 1.3882 \quad 0.00000001$

Note that, for Amber, the octahedron geometry cannot be automatically built by the "tleap" module. Therefore, an octahedron structure of the metal ion with six dummy atoms must be built in the system before the two files are loaded. The orientation of the six dummy atoms in the predefined structure can be arbitrary. The next energy minimization process will correct the orientation. 


\section{Reference}

(1) Noyes, R. M., Thermodynamics of Ion Hydration as a Measure of Effective Dielectric Properties of Water. J. Am. Chem. Soc. 1962, 84, 513-522.

(2) Rosseinsky, D., Electrode Potentials and Hydration Energies. Theories and Correlations. Chem. Rev. 1965, 65, 467-490.

(3) Marcus, Y., A Simple Empirical Model Describing the Thermodynamics of Hydration of Ions of Widely Varying Charges, Sizes, and Shapes. Biophys. Chem. 1994, 51, 111-127.

(4) Marcus, Y., Ionic Radii in Aqueous Solutions. Chem. Rev. 1988, 88, 1475-1498.

(5) Smirnov, P.; Trostin, V., Structural Parameters of Close Surroundings of Sr2+ and Ba2+ Ions in Aqueous Solutions of Their Salts. Russ. J. Gen. Chem. 2011, 81, 282-289.

(6) Miyanaga, T.; Watanabe, I.; Ikeda, S., Amplitude in EXAFS and Ligand Exchange Reaction of Hydrated 3d Transition Metal Complexes. Chem. Lett. 1988, 17, 1073-1076.

(7) Ohtaki, H.; Radnai, T., Structure and Dynamics of Hydrated Ions. Chem. Rev. 1993, 93, 1157-1204.

(8) He, M. M.; Clugston, S. L.; Honek, J. F.; Matthews, B. W., Determination of the Structure of Escherichia coli Glyoxalase I Suggests a Structural Basis for Differential Metal Activation. Biochemistry 2000, 39, 8719-8727.

(9) Duarte, F.; Bauer, P.; Barrozo, A.; Amrein, B. A.; Purg, M.; Åqvist, J.; Kamerlin, S. C. L., Force Field Independent Metal Parameters Using a Nonbonded Dummy Model. J. Phys. Chem. B 2014, 118, 4351-4362.

(10) Li, P.; Merz Jr, K. M., Taking into Account the Ion-Induced Dipole Interaction in the Nonbonded Model of Ions. J. Chem. Theory Comput. 2013, 10, 289-297. 